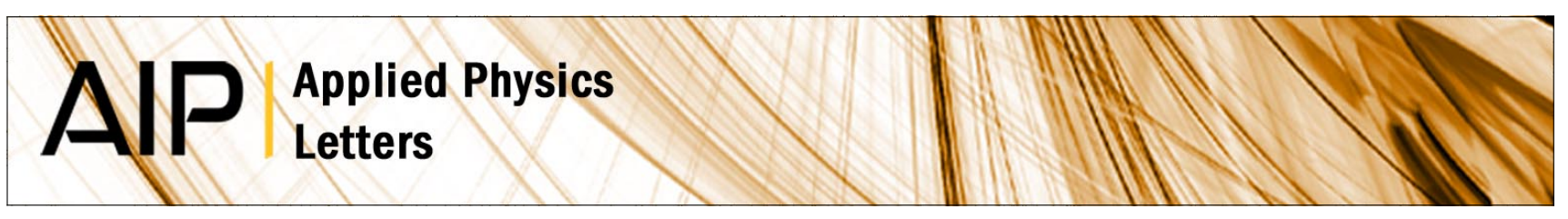

\title{
Hysteresis loops of ferroelectric bilayers and superlattices
}

Khian-Hooi Chew, Lye-Hock Ong, Junaidah Osman, and D. R. Tilley

Citation: Appl. Phys. Lett. 77, 2755 (2000); doi: 10.1063/1.1320040

View online: http://dx.doi.org/10.1063/1.1320040

View Table of Contents: http://apl.aip.org/resource/1/APPLAB/v77/i17

Published by the AIP Publishing LLC.

Additional information on Appl. Phys. Lett.

Journal Homepage: http://apl.aip.org/

Journal Information: http://apl.aip.org/about/about_the_journal

Top downloads: http://apl.aip.org/features/most_downloaded

Information for Authors: http://apl.aip.org/authors

\section{ADVERTISEMENT}
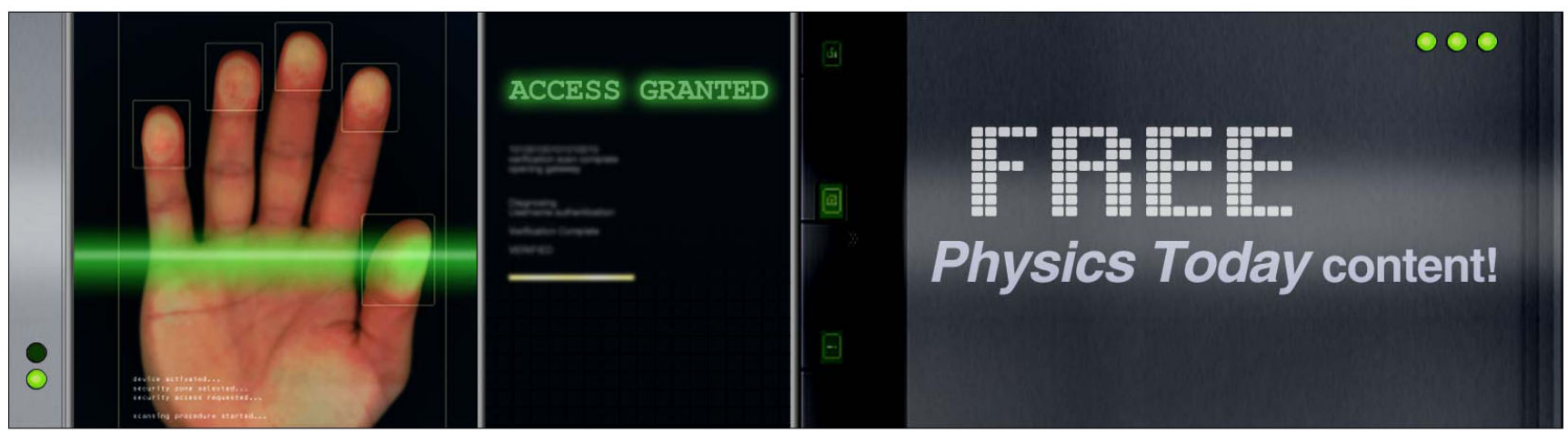


\title{
Hysteresis loops of ferroelectric bilayers and superlattices
}

\author{
Khian-Hooi Chew, Lye-Hock Ong, Junaidah Osman, ${ }^{\text {a) }}$ and D. R. Tilley \\ School of Physics, Universiti Sains Malaysia, 11800 USM, Penang, Malaysia
}

(Received 13 March 2000; accepted for publication 29 August 2000)

\begin{abstract}
A ferroelectric superlattice with an antiferroelectric interfacial coupling is considered; the same model describes a bilayer with antiferroelectric coupling. By mapping minimum points in the Landau free energy expression and plotting them against the applied electric field, a triple hysteresis loop pattern is obtained. The loop patterns vary between typically ferroelectric and typically antiferroelectric depending on the layer thicknesses and the magnitude of the interfacial-coupling constant. This work suggests the possibility of designing multilayer elements for computer memories with four or more different storage states. (c) 2000 American Institute of Physics.
\end{abstract}

[S0003-6951(00)03843-2]

Size effects are important in ferroelectric (FE) materials since they are systems with long-range order. Indeed, FE films and superlattices are usually found to have different physical properties from bulk ferroelectrics. For example, the dielectric constants of multilayered FE thin films are dependent on layer thicknesses ${ }^{1}$ and it has been demonstrated that the oscillation of the pinned domain walls in an external electric field yielded a giant dielectric constant in an epitaxial FE superlattice. ${ }^{2}$ More recently, soft-mode hardening has been observed in $\mathrm{SrTiO}_{3}$ films. ${ }^{3,4}$ Raman-scattering observation of confined transverse optical phonons in $\mathrm{PbTiO}_{3} / \mathrm{BaTiO}_{3}$ superlattices ${ }^{5}$ is an indication of high growth quality since confinement depends on sharp interfaces. A recent letter ${ }^{6}$ reports the observation of the full intrinsic hysteresis loop in very thin Langmuir-Blodgett FE films. Taken as a whole, these and other results imply an important role played by interfacial coupling in FE films and superlattices.

A substantial amount of theoretical work on FE films has been reported $^{7,8}$ and a small amount on superlattices. In films, attention has been focused on spatial variation of the polarization near surfaces and interfaces, which can arise for a variety of reasons, and on the effect of the depolarization field which arises when the applied field and polarization are normal to the film. A general formulation including both these effects is available. ${ }^{8,9}$ For superlattices, Qu et al. ${ }^{10}$ have predicted a size-driven phase transition which is dependent on the strength of the interfacial coupling and the layer thicknesses. Ma et al. ${ }^{11}$ have addressed the effect of antiferroelectric coupling on the spontaneous polarization in a superlattice consisting of two coupled FE sublattices. They have shown numerically that in the absence of an applied field the magnitude of the coupling enhances the antiferroelectric behavior in the structure when both sublattices are the same in thickness and composition.

The application of FE thin films in computer memories ${ }^{12}$ has increased the interest in studying polarization reversal, ${ }^{10,12-14}$ which can be characterized by the dielectric hysteresis loop. It is of value to study means by which systems can be designed to have specified hysteresis loops. We

${ }^{a}$ Electronic mail: junaidah@usm.my consider here a bilayer of two ferroelectric films with an antiferroelectric coupling across the interface and also a superlattice of ferroelectric layers with this coupling. We show that by choice of the layer thicknesses the hysteresis loops can be varied over a very wide range. Our work is speculative in that we do not say how such a coupling might be produced in practice. In our view, the great importance of the analogous magnetic coupling in giant-magnetoresistance devices ${ }^{15}$ justifies some degree of speculation. Because this is a preliminary study, we take the simplest possible model; we shall argue that the essential results are not an artifact of the simplifications.

It will be recalled that ferroelectrics have simple hysteresis loops ${ }^{6,14,16}$ whereas the hysteresis for an antiferroelectric material consists of double loops. ${ }^{16,17}$ Our point is basically that in the system we propose the hysteresis loop can be designed to any form between these extremes. Our analysis is similar to that applied by Ricinschi et al. ${ }^{14}$ for bulk ferroelectrics.

We consider the two systems sketched in Fig. 1, that is a bilayer of materials 1 and 2 [Fig. 1(a)] or a superlattice 1/2/ $1 / 2 / \ldots$. Fig. 1(b)] with layer thicknesses $L_{1}$ and $L_{2}$. Layers 1 and 2 are $\mathrm{FE}$ with an antiferroelectric interface coupling. Since we concentrate on the effect of the interface coupling, we make the simplest assumption, namely that $P$ is constant within each layer. With these assumptions, the free energy per unit area (and per unit cell in a superlattice) $F$ involves just two scalar variables $P_{1}$ and $P_{2}$, and we write

$$
\begin{aligned}
F= & \frac{A_{1}}{2 \epsilon_{0}} P_{1}^{2} L_{1}+\frac{B_{1}}{4 \epsilon_{0}^{2}} P_{1}^{4} L_{1}-P_{1} E L_{1}+\frac{A_{2}}{2 \epsilon_{0}} P_{2}^{2} L_{2} \\
& +\frac{B_{2}}{4 \epsilon_{0}^{2}} P_{2}^{4} L_{2}-P_{2} E L_{2}+J P_{1} P_{2} .
\end{aligned}
$$

For a bilayer, $P_{1}$ and $P_{2}$ are the polarizations in the two layers and for a superlattice they apply to any of the layers 1 and 2. The first three terms are the standard free energy density for material 1 , including the coupling to the applied field $E$ and multiplied by the layer thickness $L_{1}$. We assume that $A_{1}$ is temperature dependent, $A_{1}=a_{1}\left(T-T_{C 1}\right)$ with critical temperature $T_{C 1}$, and that $B_{1}$ is independent of temperature and positive. The next three terms are the same 


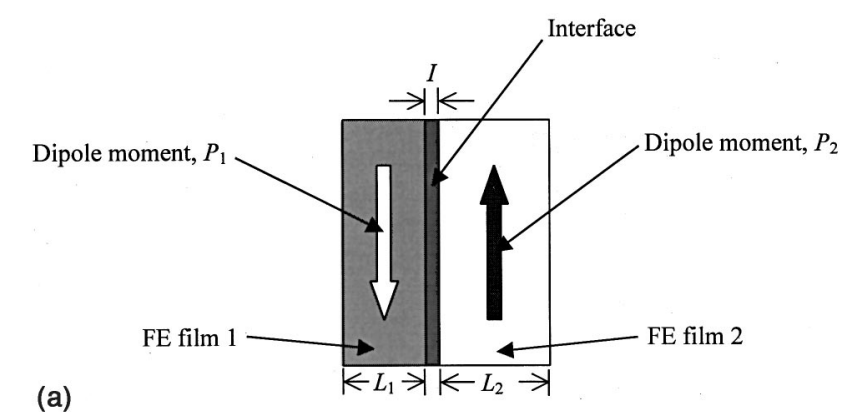

(a)

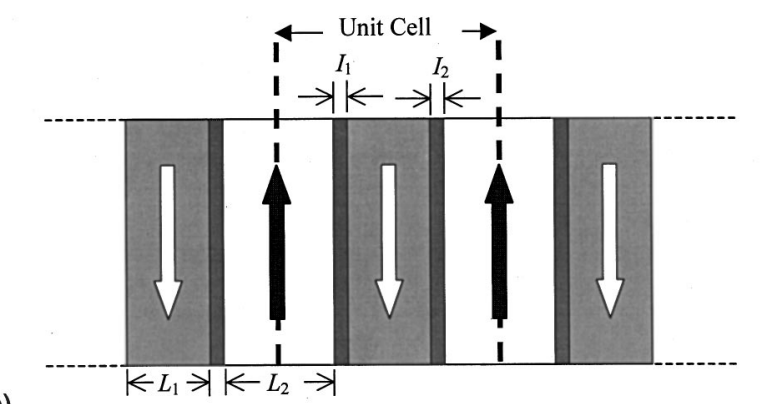

(b)

FIG. 1. Systems considered here, (a) bilayer and (b) superlattice. In the absence of a field, the polarizations are aligned in opposite directions.

thing for layer 2. The final term in (1) describes interface coupling with coupling constant $J$. For a superlattice, $J$ includes two terms from the two interfaces $I_{1}$ and $I_{2}$ in the unit cell marked in Fig. 1(b), and for a bilayer it describes the single interface $I$. In both cases, we assume $J>0$ which favors antiferroelectric coupling in which $P_{1}$ and $P_{2}$ are in opposite directions, as marked in Fig. 1.

The limitations of (1) are these. First, we are assuming that $P_{1}$ and $P_{2}$ are constant in each layer, thus ignoring possible spatial variation within each layer. Second, depolarization is not included. We can look at this two ways: either we assume that $P_{1}$ and $P_{2}$ and the applied field $E$ lie parallel to the interfaces or we take them perpendicular (as they are in practical devices) and regard depolarization as an effect to be included later in a more realistic study. We prefer the latter view. Third, the free energy of each film is written for a second-order transition although most ferroelectrics have first-order transitions. All of these restrictions could be removed. As remarked, a free-energy expression including spatial variation and depolarization effects is available ${ }^{8,9}$ and this could easily be generalized to the present case, including first-order transitions. A recently proposed numerical scheme $^{18}$ is adequate to study the full expression.

In the absence of the applied field $E$ the ground state of (1) has $P_{1}$ and $P_{2}$ in opposite directions, $P_{1} P_{2}<0$, since this minimizes the coupling energy; the zero-field equilibrium directions of $P_{1}$ and $P_{2}$ are shown schematically in Fig. 1. An important implication is that even if films 1 and 2 are made of the same ferroelectric material the bilayer is not the same as a single film of thickness $L_{1}+L_{2}$ because the interfacial coupling ensures that for small field the polarization directions are opposite in the two films. A magnetic analog would be two Fe films separated by a thin $\mathrm{Cr}$ layer that gives antiferromagnetic coupling; the basis of giantmagnetoresistance devices is that in the absence of an applied field the Fe moments are in opposite directions. ${ }^{19}$ Since
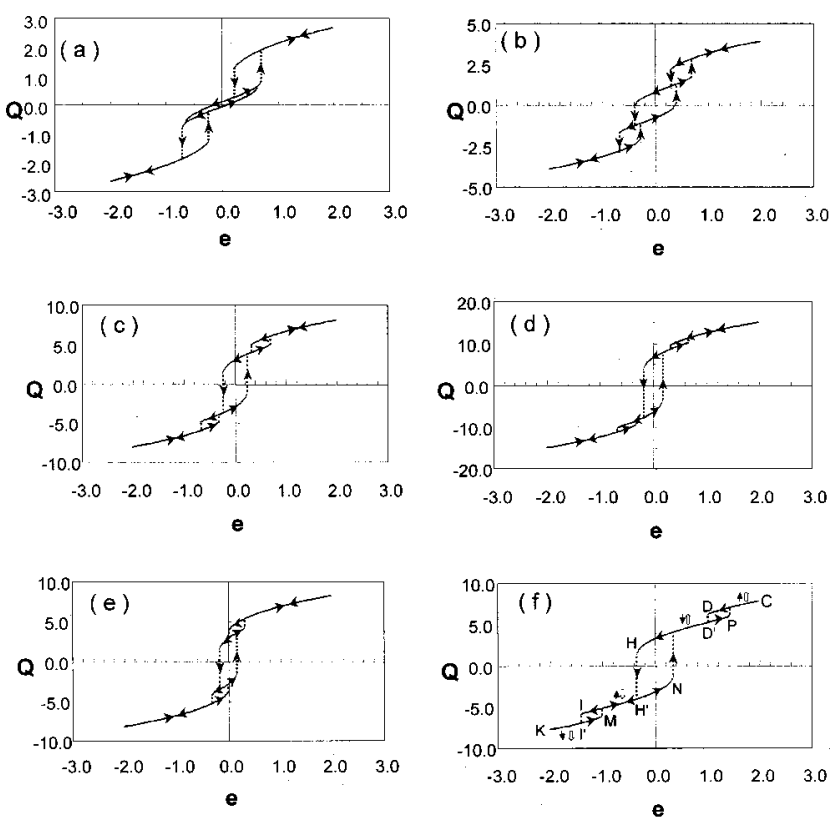

FIG. 2. Plots of $p_{1}+p_{2} \ell$ (denoted $Q$ ) vs $e$ for $t=0.5$ : (a) $j=0.5, \ell=1.1$, (b) $j=0.5, \ell=2.0$, (c) $j=0.5, \ell=5.0$, (d) $j=0.5, \ell=10.0$, (e) $j=0.2$, $\ell=5.0$, and (f) $j=1.0, \ell=5$.0. In Fig. 2(f) symbols $\uparrow$ and $\downarrow$ represent up and down polarization for $p_{1}$ (layer 1) and symbols $\Uparrow$ and $\Downarrow$ represent up and down polarization for $p_{2}$ (layer 2).

our aim is to study the simplest possible model we therefore take 1 and 2 as identical media so that $A_{1}=A_{2}=a\left(T-T_{c}\right)$ and $B_{1}=B_{2}>0$. In dimensionless quantities, the free energy expression in (1) now becomes

$$
\begin{aligned}
f= & \frac{(t-1)}{2}\left(p_{1}^{2}+\ell p_{2}^{2}\right)+\frac{1}{4}\left(p_{1}^{4}+\ell p_{2}^{4}\right)-e\left(p_{1}+\ell p_{2}\right) \\
& +j p_{1} p_{2},
\end{aligned}
$$

where $f=\epsilon_{0}^{2} F / B L_{1}, \quad \ell=L_{2} / L_{1}, \quad t=T / T_{c}, \quad j=J / L_{1}, \quad e$ $=\left(L_{1} E / a T_{c}\right)\left(\epsilon_{0} B / a T_{c}\right)^{1 / 2}$ and $p_{i}^{2}=\left(\epsilon_{0} a T_{c} / B\right) P_{i}^{2}$ (with $i=1$ or 2). The dimensionless free energy, $f$, is a function of two variables, $p_{1}$ and $p_{2}$ and we now investigate the implications of (2) since detailed study of $p_{1}$ and $p_{2}$ variations as $e$ changes provides a complete picture of polarization reversal.

For a given value of $e$ the equilibrium values of $p_{1}$ and $p_{2}$ are those that give minima of $f$ so that they are solutions of

$$
\begin{aligned}
& j p_{2}-e+(t-1) p_{1}+p_{1}^{3}=0, \\
& j p_{1}-e \ell+(t-1) \ell p_{2}+\ell p_{2}^{3}=0
\end{aligned}
$$

and to obtain the hysteresis loop pattern we plot $p_{1}+p_{2} \ell$ vs $e$. Before showing results we note that at points of inflexion $\left(\partial^{2} f / \partial p_{1}^{2}\right)$ and $\left(\partial^{2} f / \partial p_{2}^{2}\right)$ are both zero; these conditions give $p_{1}=p_{2}=[(1-t) / 3]^{1 / 2}$ at a coercive field

$$
e_{c}=[j+(t-1)]\left(\frac{1-t}{3}\right)^{1 / 2}+\left(\frac{1-t}{3}\right)^{3 / 2} .
$$

This gives extreme points in the hysteresis loops where minima, maxima, and inflexion points in $f$ coincide; these represent the onset of polarization reversal.

Figure 2 shows typical hysteresis plots calculated from numerical solutions of (3). In making these plots, we selected only solutions that correspond to local minima of $f$. The 
temperature $t$ is not very crucial for qualitative features so we maintain $t=0.5$ and display plots for various values of coupling $j$ and thickness ratio $\ell$. In Figs. 2(a)-2(d) the value $j=0.5$ is maintained and $\ell$ is varied. The hysteresis loop pattern begins with large double loops typical of an antiferroelectric since $\ell$ is close to 1.0 (i.e., layers 1 and 2 are about the same thickness). However, as $\ell$ increases in value a central loop opens up so that triple loops appear when 1 and 2 are sufficiently different in thickness [e.g., Fig. 2(b) for $\ell=2.0]$. Further increase in $\ell$, for example in the sequence $\ell=2.0$ [Fig. 2(b)] to $\ell=5.0$ [Fig. 2(c)] and $\ell=10.0$ [Fig. $2(\mathrm{~d})]$, widens the size of the central loop and reduces the outer loops vertically. In this sequence the system is becoming more ferroelectric with the outer loops for large $l$ showing vestigial antiferroelectricity. The effect of the coupling constant, $j$, on the loop pattern can be interpreted when Figs. 2(c), 2(e), and 2(f) are compared. In these figures the value $\ell=5.0$ is fixed and $j$ values in increasing order [Fig. 2(e) with $j=0.2$, Fig. 2(c) $j=0.5$, and Fig. 2(f) $j=1.0$ ], are used. As $j$ increases in value the three loops stretch further out horizontally.

The switching behavior can be understood from Fig. 2(f), for example. Suppose we begin at point $C$ and decrease $e$. To achieve a minimum in $f, j$ favors $p_{1}$ and $p_{2}$ to be opposite in sign, whereas $e$ favors $p_{1}$ and $p_{2}$ aligned with the field. In region $\mathrm{CD}$ the value of $e$ is large and it has a stronger influence on $p_{1}$ and $p_{2}$ than $j$ does so that both $p_{1}$ and $p_{2}$ are switched up. Point D represents the onset of polarization reversal, so as $e$ is decreased further in value the influence of $j$ begins to predominate over $e$. Since layer 1 occupies a smaller volume, $p_{1}$ switches down and $p_{2}$ stays up. Thus along $\mathrm{D}^{\prime} \mathrm{H} p_{1}$ and $p_{2}$ are oppositely polarized. Another switching takes place at point H. Here $e$ is negative but small in magnitude and the influence of $j$ is still stronger. Since $e$ is negative, $p_{2}$ (with a larger volume) switches down and $p_{1}$ switches up. This is maintained until point I. From here $e$ is large and negative, dominating $j$ so along line $\mathrm{I}^{\prime} \mathrm{K}$ both $p_{1}$ and $p_{2}$ are switched down. If on the other hand we begin at $\mathrm{K}$ and increase $e$, the reverse sequence occurs with the degrees of hysteresis shown.

Our basic result, as just discussed, is that the four arrangements $(\uparrow \Uparrow),(\uparrow \downarrow),(\downarrow \Uparrow)$, and $(\downarrow \downarrow)$ can all occur at different part of the hysteresis loop. The reasons for this are simple and we surmize that the basic qualitative result would persist in more complicated and realistic studies. As pointed out in our first discussion of (1) and (2), in the low-field region $p_{1}$ and $p_{2}$ are driven by the interfacial coupling to be in opposite directions. We can now add that the configuration can be either $(\uparrow \downarrow)$ or $(\downarrow \Uparrow)$ depending on the way in which the lowfield region is approached. Within the present model the details of the hysteresis loops depend in different ways on the two parameters $\ell=L_{2} / L_{1}$ and $j=J / L_{1}$, both of which are controllable through selection of the layer thicknesses. The ratio $l$ controls the balance between antiferroelectric and ferroelectric character, the former predominating when $l$ is not very different from unity. Since presumably $J$ is a material parameter, the value of $j$ and therefore the importance of the interfacial coupling varies inversely with the layer thickness $L_{1}$.

We remark finally that for some values of $\ell$ and $j$, as in Figs. 2(c), 2(d), and 2(f), all four arrangements of $p_{1}$ and $p_{2}$ occur in nonhysteretic regions which may represent four possible data recording points. An obvious extension is to multilayers, so that for example a trilayer or a superlattice with a three-component unit cell may be designed to have $2^{3}=8$ recording points and so on. In particular, it should be possible to design multilayers with a sufficient number of recording points to support octal logic.

We have assumed implicitly that sufficient anisotropy is present to maintain the polarization directions along one axis. In some cases, it is possible that $P_{1}$ and $P_{2}$ may move out of alignment, so as to produce a configuration like the antiferromagnetic spin-flop state for example. This possibility requires further analysis.

The authors are grateful to Professor J. F. Scott for helpful comments. This work is supported by IRPA (Malaysian Government) Grant No. 09-02-05-6001.

${ }^{1}$ H. Tabata, H. Tanaka, and T. Kawai, Appl. Phys. Lett. 65, 1970 (1994).

${ }^{2}$ A. Erbil, Y. Kim, and R. A. Gerhardt, Phys. Rev. Lett. 77, 1628 (1996).

${ }^{3}$ A. A. Sirenko, C. Bernhard, A. Golnik, A. M. Clark, J. H. Hao, W. D. Si, and X. X. Xi, Nature (London) 404, 373 (2000).

${ }^{4}$ I. A. Akimov, A. A. Sirenko, A. M. Clark, J. H. Hao, and X. X. Xi, Phys. Rev. Lett. 84, 4625 (2000).

${ }^{5}$ F. Le Marrec, R. Farhi, M. El Marssi, J. L. Dellis, M. G. Karkut, and D. Ariosa, Phys. Rev. B 61, R6447 (2000).

${ }^{6}$ S. Ducharme, V. M. Fridkin, A. V. Bune, S. P. Palto, L. M. Blinov, N. N. Pethukova, and S. G. Yudin, Phys. Rev. Lett. 84, 175 (2000).

${ }^{7}$ W. L. Zhong, Y. G. Wang, and P. L. Zhang, Ferroelectr. Rev. 1, 131 (1998).

${ }^{8}$ D. R. Tilley, in Ferroelectric Thin Films, edited by C. Paz de Araujo, J. F. Scott, and G. W. Taylor (Gordon and Breach, Amsterdam, 1996), p. 11.

${ }^{9}$ D. R. Tilley, in Ferroelectric Ceramics, edited by N. Setter and E. L. Colla (Birkhäuser, Basel, 1993), p. 163.

${ }^{10}$ B. D. Qu, W. L. Zhong, and R. H. Prince, Phys. Rev. B 55, 11218 (1997).

${ }^{11}$ Y. Ma, X. Xu, and J. Shen, Solid State Commun. 114, 461 (2000).

${ }^{12}$ J. F. Scott, Ferroelectr. Rev. 1, 1 (1998).

${ }^{13}$ O. Auciello, J. F. Scott, and R. Ramesh, Phys. Today July, 22 (1998).

${ }^{14}$ D. Ricinschi, C. Harnagae, C. Ppusoi, L. Mitoseriut, V. Tura, and M. Okuyama, J. Phys.: Condens. Matter 10, 477 (1998).

${ }^{15}$ A. Fert, P. Grünberg, A. Barthélémy, F. Petroff, and W. Zinn, J. Magn. Magn. Mater. 140-144, 1 (1995).

${ }^{16}$ M. E. Lines and A. M. Glass, Principles and Applications of Ferroelectrics and Related Materials (Clarendon, London, 1977), pp. 81-86.

${ }^{17}$ P. Ayyub, S. Chattopadhyay, R. Pinto, and M. S. Multani, Phys. Rev. B 57, R5559 (1998).

${ }^{18}$ E. K. Tan, J. Osman, and D. R. Tilley, Solid State Commun. 116, 61 (2000).

${ }^{19}$ R. L. Comstock, Introduction to Magnetism and Magnetic Recording (Wiley, New York, 1999). 